\title{
A proof of the completeness of Lamb modes
}

\author{
Jean-Luc Akian ${ }^{1}$
}

${ }^{1}$ ONERA

April 6, 2021

\begin{abstract}
The aim of this paper is to give a precise proof of the completeness of Lamb modes and associated modes. This proof is relatively simple and short but relies on two powerful mathematical theorems. The first one is a theorem on elliptic systems with a parameter due to Agranovich and Vishik. The second one is a theorem due to Locker which gives a criterion to show the completeness of the set of generalized eigenvectors of a Hilbert-Schmidt discrete operator.
\end{abstract}

\section{Hosted file}

Akian_Lamb_Modes.pdf available at https://authorea.com/users/406052/articles/516872-a-proofof-the-completeness-of-lamb-modes 